\title{
Does Meditation Reduce Pain through a Unique Neural Mechanism?
}

\author{
Tim V. Salomons ${ }^{1}$ and Aaron Kucyi ${ }^{1,2}$ \\ ${ }^{1}$ Division of Brain, Imaging and Behaviour-Systems Neuroscience, Toronto Western Research Institute, Toronto, Ontario, Canada M5T 2S8, and \\ 2Institute of Medical Science, University of Toronto, Toronto, Ontario, Canada M5S 1A8 \\ Review of Zeidan et al.
}

The neuroscience of meditative practice has attracted considerable attention, in part because it represents a unique juxtaposition of Western scientific practice and Eastern mystical tradition. Beyond cultural interest, however, lies the question of whether such study has clinical/therapeutic relevance. Pain research is an ideal testing ground for this question. Many chronic pain disorders are frustratingly resistant to treatments aimed at eliminating the sensory component of pain, prompting increased focus on reducing suffering by altering emotional and evaluative components of pain. Mindfulness-based meditative practices, which aim to focus attention on present experiences while reducing evaluative and affective responses, appear tailor-made for this task.

Several studies have demonstrated beneficial effects of mindfulness-based interventions for both chronic and acute pain. Most of these have demonstrated effects of mindfulness on the affective and evaluative components of pain (McCracken et al., 2007; Morone et al., 2008; Perlman et al., 2010),

Received June 7, 2011; revised July 20, 2011; accepted July 21, 2011.

This work was supported by the University of Toronto Centre for the Study of Pain (Clinician-Scientist award to T.V.S.) and Canadian Institutes of Health Research (Master's Award to A.K.). We thank Karen Davis, Donal Maccoon, and Joshua Grant for comments on the manuscript.

Correspondence should be addressed to Dr. Tim V. Salomons, Division of Brain, Imaging and Behaviour-Systems Neuroscience, Toronto Western Research Institute, Toronto Western Hospital, University Health Network, 399 Bathurst Street, Room MP14-303, Toronto, Ontario, Canada M5T 258. E-mail: tvsalomons@gmail.com.

DOI:10.1523/JNEUROSCI.2843-11.2011

Copyright $\odot 2011$ the authors $\quad 0270-6474 / 11 / 3112705-03 \$ 15.00 / 0$ but some have also demonstrated effects on sensory thresholds (Grant et al., 2010, 2011) and perceived pain intensity (Grant and Rainville, 2009). Exploration of the biological mechanisms of such treatments may increase our ability to treat pain disorders by enhancing our understanding of endogenous pain regulation.

Recent studies of healthy long-term meditation practitioners suggest that extensive mental training can result in thickening of cortical regions associated with pain processing, including midcingulate cortex (MCC) and primary and secondary somatosensory cortices (Grant et al., 2010). A study of event-related potentials during anticipation of pain found that long-term meditation practice alters anticipatory evaluation and processing of pain in MCC and other pain regions (Brown and Jones, 2010). Functional imaging demonstrated that during pain, practitioners had reduced activation in amygdala, hippocampus, and emotional/ evaluative regions of prefrontal cortex, as well as increased activation in the MCC, thalamus, and insula (Grant et al., 2011). Additionally, altered pain sensitivity was associated with reduced functional connectivity between MCC and dlPFC. Grant et al. (2011) interpreted these findings as consistent with the meditative goals of increasing attention to present sensory experience (in this case, pain) while reducing emotional and evaluative responses.

Findings from long-term practitioners are of enormous theoretical interest as they demonstrate that intensive mental training can result in clinically beneficial neuroplasticity. Nevertheless, the level of training of the practitioners studied is beyond the scope of what many individuals with clinical pain conditions might be able to achieve, leading to questions of whether brief mindfulness-based interventions might also alter the way that pain is processed and experienced.

In this respect, a recent study in The Journal of Neuroscience by Zeidan et al. (2011) is an important step forward. Zeidan et al. (2011) scanned 15 healthy volunteers with pulsed arterial spin labeling (ASL), an MRI pulse sequence that provides a quantitative measure of cerebral blood flow (CBF) using water as a flow tracer. Scan sessions occurred before and after a brief mindfulness-based training intervention consisting of four $20 \mathrm{~min}$ sessions on successive days. In the first (pretraining) scan, subjects were stimulated with noxious $\left(49^{\circ} \mathrm{C}\right)$ or innocuous $\left(35^{\circ} \mathrm{C}\right)$ heat in both an attention-neutral and an attention-to-breath (ATB) control condition. Pain intensity and unpleasantness ratings were taken after each series of noxious heat delivery. The second (posttraining) scan session was identical to the first except that subjects' ability to focus on their breathing sensations while reducing distractions, judgments, and emotional responses had presumably been enhanced by mindfulness training. Thus, the ATB condition pretraining and the meditation condition posttraining were 
matched in terms of instructions and divided attention (to breath) but differed in terms of the individual's ability to achieve a nonjudgmental stance toward the pain stimulus.

Zeidan et al. (2011) reported significant changes from pretraining to posttraining (ATB vs meditation) in average pain intensity and unpleasantness ratings across subjects $[\sim 40 \%$ and $57 \%$ reductions, respectively, as inferred from the similarity observed in their Fig. 2 between ratings for the rest condition posttraining and the ATB condition pretraining]. Within the second (posttraining) MRI session, significant reductions in pain-induced CBF were found in the contralateral primary somatosensory cortex during meditation relative to the attention-neutral condition. Training-induced reductions in pain intensity were correlated with meditationinduced activity (main effect including meditation with and without painful stimulation) in the right anterior insula and MCC. Training-induced reductions in pain unpleasantness were correlated with orbitofrontal cortex (OFC) activation and thalamic deactivation during meditation.

The use of ASL is a notable feature of Zeidan et al.'s (2011) study. Although ASL lacks the spatial and temporal resolution of BOLD fMRI, it is well suited for examining the neural mechanisms through which meditation might affect clinical pain conditions. One reason is that because the ASL signal is sensitized to perfusion rather than blood oxygenation, it is not susceptible to slow-drift artifacts that are present in BOLD fMRI experiments when stimulus/task epochs exceed $1 \mathrm{~min}$. This makes ASL ideal for studying both chronic pain and meditation, which involve sustained neural activity. The recent application of ASL to monitor dynamic changes in CBF over several minutes in a muscular pain model (Owen et al., 2010) highlights the suitability of ASL for examining naturally occurring pain states. The study by Zeidan and colleagues (2011) demonstrates advantageous use of ASL for examining meditation, as the authors were able to use longer functional epochs (e.g., $5 \mathrm{~min}, 55 \mathrm{~s}$ blocks of thermal stimulation) to examine a sustained meditative state. Together, these studies suggest that examining the effects of a sustained meditative state on naturally occurring pain would be a promising and clinically relevant future application of ASL.

Zeidan et al.'s (2011) study increases our understanding of the neural mechanisms through which short-term meditative training can affect the pain experience. The authors suggest that meditation alters pain through multiple mechanisms, some of which might be common to other forms of cognitive modulation of pain (e.g., reappraisal, perceived control, expectation of relief, etc.). Precise characterization of the cognitive and biological mechanisms through which mindfulness uniquely alters pain is a critical challenge for future research.

A cognitive mechanism that is thought to be unique to mindfulness is the combination of increased attention and reduced negative evaluation. As anterior insula and MCC play a role in salience detection and motor preparedness (Menon and Uddin, 2010), increased activation in these regions may represent a neural substrate of increased attention to perceptually salient features of pain. Given that increased attention to pain and accompanying activation in these two regions is more commonly associated with increased experience of pain, the finding that activation in these regions is associated with reduced pain intensity appears counterintuitive, but it is consistent with the findings of Grant and colleagues (2011) in experienced meditators.

As increased attention and activation in MCC and insula would be expected to increase the pain experience, the key to reported analgesic effects of meditation training might be the co-occurring reduction in emotional and evaluative responses. Thus it is noteworthy that both Zeidan et al. (2011) and Grant et al. (2011) found activation patterns in regions associated with downregulation of negative affective responses (Ochsner and Gross, 2005). Grant et al. (2011) found functional decoupling of dlPFC and cingulate, which they attributed to dissociation between attention to pain and evaluation of pain. Zeidan and colleagues (2011) noted an inverse correlation between OFC activation and unpleasantness ratings, which was attributed to altered processing of reward and hedonic experiences. The degree of concordance between these studies suggests that meditative practices may indeed reduce pain through a unique neural mechanism, one corresponding to increased attention and reduced evaluative/emotional responses.

Although these patterns of activation appear consistent with the hypothesized cognitive mechanisms of mindfulness, future work must verify these mechanisms empirically. Cognitive benefits of mindfulness have been demonstrated (Lutz et al., 2008), but it should not be assumed that these effects occur in all populations or that they are the mechanism through which meditative training affects pain perception. For instance, it is noteworthy that Zeidan and colleagues (2011) observed an $\sim 57 \%$ decrease in unpleasantness ratings following meditative training but only a $14 \%$ increase in self-reported mindfulness. While psychometric factors could explain this discrepancy, it clearly cannot be taken for granted that changes in pain experience can be attributed solely to mindfulness. Coupling pain with well established cognitive tasks that manipulate and measure attention and affective responses to pain is necessary to link changes in self-report and neural activation with the purported cognitive mechanisms of mindfulness training.

A shortcoming of the Zeidan et al. (2011) study in terms of testing the mechanistic specificity of mindfulness is the lack of an active-treatment control group. There has been considerable debate as to whether the benefits of particular psychotherapeutic interventions result from cognitive factors specific to those therapies or to factors, such as expectation of efficacy, that are characteristic of all effective treatments (Wampold et al., 1997). As demonstrated by studies of placebo analgesia, strong belief in the stated benefits of a treatment such as mindfulness may have direct benefits on the experience and processing of pain (Wiech et al., 2008). Furthermore, such beliefs may result in unconscious biases toward self-report consistent with these stated benefits (e.g., reductions in unpleasantness ratings). Comparing mindfulness with psychotherapeutic interventions that do not contain a mindfulness component but are otherwise matched for common factors such as degree of patient engagement and efficacy beliefs would provide a more precise understanding of the degree to which the mechanisms of mindfulness are distinct from other forms of cognitive modulation of pain.

In conclusion, Zeidan et al. (2011) provide important insights into the neural mechanisms through which meditation alters the pain experience. The study is particularly promising from the standpoint of clinical neuroscience, as the observed effects are due to clinically plausible, short-term meditation training. As both meditation and chronic pain present challenges for traditional functional neuroimaging, the use of ASL in the present study is also highly promising. As the scientific investigation of meditative practice continues to expand, however, a key challenge will be determining the degree to 
which changes in both the experience of pain and associated neural processing are due to cognitive and biological mechanisms specific to mindfulness.

\section{References}

Brown CA, Jones AK (2010) Meditation experience predicts less negative appraisal of pain: electrophysiological evidence for the involvement of anticipatory neural responses. Pain 150:428-438.

Grant JA, Rainville P (2009) Pain sensitivity and analgesic effects of mindful states in Zen meditators: a cross-sectional study. Psychosom Med 71:106-114.

Grant JA, Courtemanche J, Duerden EG, Duncan GH, Rainville P (2010) Cortical thickness and pain sensitivity in Zen meditators. Emotion 10:43-53.

Grant JA, Courtemanche J, Rainville P (2011) A non-elaborative mental stance and decou- pling of executive and pain-related cortices predicts low pain sensitivity in Zen meditators. Pain 152:150-156.

Lutz A, Slagter HA, Dunne JD, Davidson RJ (2008) Attention regulation and monitoring in meditation. Trends Cogn Sci 12:163-169.

McCracken LM, Gauntlett-Gilbert J, Vowles KE (2007) The role of mindfulness in a contextual cognitive-behavioral analysis of chronic pain-related suffering and disability. Pain 131:63-69.

Menon V, Uddin LQ (2010) Saliency, switching, attention and control: a network model of insula function. Brain Struct Funct 214:655667.

Morone NE, Greco CM, Weiner DK (2008) Mindfulness meditation for the treatment of chronic low back pain in older adults: a randomized controlled pilot study. Pain 134:310-319.

Ochsner KN, Gross JJ (2005) The cognitive control of emotion. Trends Cogn Sci 9:242-249.

Owen DG, Clarke CF, Ganapathy S, Prato FS, St
Lawrence KS (2010) Using perfusion MRI to measure the dynamic changes in neural activation associated with tonic muscular pain. Pain 148:375-386.

Perlman DM, Salomons TV, Davidson RJ, Lutz A (2010) Differential effects on pain intensity and unpleasantness of two meditation practices. Emotion 10:65-71.

Wampold BE, Mondin GW, Moody M, Stich F, Benson K, Ahn H (1997) A meta-analysis of outcome studies comparing bona fide psychotherapies: empirically, "all must have prizes." Psychol Bull 122:203-215.

Wiech K, Ploner M, Tracey I (2008) Neurocognitive aspects of pain perception. Trends Cogn Sci 12:306-313.

Zeidan F, Martucci KT, Kraft RA, Gordon NS, McHaffie JG, Coghill RC (2011) Brain mechanisms supporting the modulation of pain by mindfulness meditation. J Neurosci 31:5540-5548. 